\title{
Comparison of Fusarium Head Blight Resistance in Cytoplasmic Male Sterile, Maintainer and Restorer Lines in Winter Wheat
}

\author{
M.Y. WANG ${ }^{1}$, P.S. BAENZIGER ${ }^{2 *}$, I.S. El-BASYONI ${ }^{3}$ and S.N. WeGUlO ${ }^{4}$ \\ ${ }^{1}$ Department of Agronomy, Northwest A\&F University, Shaanxi, 712100, PRC \\ ${ }^{2}$ Department of Agronomy and Horticulture, University of Nebraska-Lincoln, NE 68583, USA \\ ${ }^{3}$ Crop Sciences Department, Damanhour University, Damanhour, 22516, Egypt \\ ${ }^{4}$ Department of Plant Pathology, University of Nebraska-Lincoln, NE 68583, USA \\ (Received 23 June 2014; Accepted 8 October 2014; \\ Communicated by Á. Mesterházy and X.F. Zhang)
}

\begin{abstract}
Fusarium head blight (FHB, caused by Fusarium graminearum) is a severe disease which threatens the yield and quality of harvested grain products, and hence causes major economic losses in wheat (Triticum spp.) and other small grain cereals. One of the infection routes for the pathogen is believed to be through the extruded anther. In order to study the role of the anther on FHB infection, FHB resistance was measured among wheat (Triticum aestivum L.) cultivars with non-extruded infertile anthers in cytoplasmic male sterile (CMS) lines and with extruded fertile anthers in their alloplasmic maintainer lines, and in an unrelated restorer line. We artificially inoculated the lines with $F$. graminearum (isolate NE 90) at $1.0 \times 10^{5}$ or $2.0 \times 10^{5}$ spores $\mathrm{mL}^{-1}$ for 72 or 96 hours. The results indicated that CMS cultivars were significantly more resistant than the maintainer and restorer cultivars. As the CMS lines differed from the maintainer lines by cytoplasm, anther health, anther extrusion, and seed set, additional experiments are needed to determine the role of each factor in infection. However, this research indicates that fertile anthers most likely are an important part of the infection pathway and of susceptibility to FHB.
\end{abstract}

Keywords: extruded fertile anther, scab, alloplasmic lines

\section{Introduction}

Scab or Fusarium head blight (FHB, Fusarium spp.) remains a severe and common disease of wheat (Triticum spp. L.) and other small grains. This disease is caused by a complex of 17 species of which $F$. graminearum, F. culmorum, $F$. avenaceum, F. poae and Microdochium nivale are predominant (Parry et al. 1995; Brennan et al. 2007; cited Audenaert et al. 2009). In addition to grain yield losses, the infected grains are contaminated with the mycotoxin deoxynivalenol (DON) and are frequently shriveled. The contaminated grain with high levels of DON is toxic to livestock and humans (McMullen et al. 1997; Jones and Mirocha 1999) thus increasing the economic loss. Fusarium head blight

\footnotetext{
* Corresponding author; E-mail: pbaenziger1@unl.edu
} 
is economically one of the most serious fungal diseases of wheat in many regions of the world (Tomasovic et al. 2007). In recent years, FHB remains endemic in Europe, North America, and Asia, especially in China under favorable environmental conditions. In the Northern Great Plains of the US, an economic loss of US\$2.7 billion has been attributed to FHB over the period 1998 to 2000 (Nganje et al. 2004). Under the auspices of the US Wheat and Barley Scab Initiative (USWBSI; http//www.scabusa.org) researchers launched a major effort to reduce the economic impact of FHB on the wheat and barley (Hordeum vulgare L.) industries (Brown-Guedira et al. 2008). Since 2007, epidemics of FHB have occurred in the Central Great Plains, causing significant economic losses in hard winter wheat in the states of Kansas, Nebraska and South Dakota (USWBSI, 20072011; cited in Hernandez Nopsa et al. 2012). In 2012, approximately 1,640,000 hectares were lost due to scab which was $68.3 \%$ of the wheat acreage in the Huang-Huai Area in China. This percentage loss is the highest in China's recorded history (Dai 2012).

The breeding and cultivation of resistant wheat cultivars is the most promising strategy to reduce the risk of FHB (Groth et al. 2011). Resistance or tolerance to FHB can take many forms from nuclear resistance genes or QTLs (e.g. Fhb1) (Cuthbert et al. 2006) to mechanical resistance where the infection pathway is blocked. Wheat pathologists have been interested in the importance of anthers in FHB infection since 1933 when the first symptoms of FHB appeared on non-extruded anthers of wheat (Pugh et al. 1933). In 1971, Strange and Smith observed a prolific mycelial growth on anthers of wheat. In 1974, Strange et al. isolated and characterized betaine and choline as fungal growth stimulants resulting in prolific $F$. graminearum growth. These chemicals were found in greater concentration in the anthers than in other parts of the florets (Strange et al. 1974). Miller et al. (2004) also found that $F$. graminearum had high affinity to anthers and pollen. However, Kang and Buchenauer (2000) reported the presence or absence of anthers was not important in infection. The initial infection was not necessarily through anthers, but anthers and pollen promoted hyphal growth. Thus, the presence of anthers in florets could increase disease severity. Later studies have also highlighted the importance of anther extrusion. Kubo et al. (2010) found that cleistogamous lines initially had reduced FHB infection, but chasmogamous lines were not different for kernel shriveling or mycotoxin levels. Furthermore, Kubo et al. (2013) found minor differences in anther extrusion affected FHB resistance in wheat. In this study, again, lines with cleistogamous flowers had lower FHB symptoms, as did lines with rapid anther extrusion. However, lines with partial anther extrusion had the highest levels of FHB symptoms. In other studies, some wheat genotypes with high levels of anther extrusion tended to develop less FHB and had lower levels of DON (Skinnes et al. 2010). Liu et al. (2013) found that anther extrusion and plant height were negatively correlated with FHB severity. In summary, many researchers have reported that anthers play a critical role in FHB infection, but other research indicated there are other infection pathways including glumes (Tu 1930; cited in Kang and Buchenauer 2000), spikelets or rachis (Schroeder 1955; cited in Kang and Buchenauer 2000).

Cytoplasmic male sterility (CMS) is determined by nuclear genes interacting with the cytoplasm. The cytoplasm in wheat is maternally inherited. When CMS is present, the 
anthers are shriveled and non-functional. In 1951, Kihara first discovered CMS in wheat (Song and Hedgcoth 1993). Later, Wilson and Ross (1962a, b) discovered Triticum timopheevi Zhuk could be used for CMS and created three matched lines that were called the male sterile, maintainer, and restorer lines. The interest in CMS in wheat is that it can be used to create hybrid wheat. In hybrid seed production, the CMS line is the female and eliminates the need for hand or chemical emasculation. Cytoplasmic male sterile lines also provide a way to look at the role of anthers in FHB infection. If the anthers are one part of an essential infection pathway for $F$. graminearum, CMS lines without this infection pathway due to shriveled, non-functional and non-extruded anthers should be more resistant to FHB compared to the maintainer and restorer lines (both of which have fertile, extruded anthers). Matsui et al. (2002) studied the effect of cytoplasm and sterile anthers in barley (Hordeum vulgare L.). Barley plants in CMS cytoplasms that did not produce pollen had lower FHB damage than plants that did produce fertile pollen (e.g. restored alloplasmic near-isogenic lines). The investigators concluded that viable pollen and fertile anthers were important for FHB infection. The objective of this research was to compare FHB resistance in CMS wheat cultivars with their alloplasmic maintainer lines and with an unrelated restorer line. The results will also be important for hybrid production fields where the female line flowers are open for extended periods of time during pollination.

\section{Materials and Methods}

The study was conducted in a greenhouse at the University of Nebraska, Lincoln, Nebraska, USA. Five cultivars including two CMS lines, '77(2)' and 'Yanshi NO.9' (T. timopheevi cytoplasm); two corresponding alloplasmic maintainer lines of 77(2) and Yanshi NO.9 (T. aestivum cytoplasm, hence male fertile; hereafter referred to as 'M 77(2)' and 'M Yanshi NO.9'); and an unrelated restorer line of 'R113' (T. timpoheevi cytoplasm, but with nuclear restorer genes, hence male fertile) were used in this study. All five lines are early-maturing hard red, semi-dwarf winter wheat lines from Shaanxi, the dominant area for winter wheat in China (Zhang et al. 2009).

All five lines were seeded and allowed to grow as seedlings after which they were vernalized for 6 weeks at $7^{\circ} \mathrm{C}$ with 12 hours of light and 12 hours of dark before being transplanted into $15 \mathrm{~cm}$ pots. Each pot was considered as one replication. The transplanted plants grew until flowering. F. graminearum (isolate NE 90) inoculum was increased on potato dextrose agar (PDA) medium. Spores were quantified using a hemocytometer, adjusted to the final concentration of $1.0 \times 10^{5}$ spores $\mathrm{mL}^{-1}$, and kept in a $50 \mathrm{~mL}$ Falcon tube at $4{ }^{\circ} \mathrm{C}$ until needed for inoculation (Hernandez Nopsa et al. 2014). Spray inoculations were performed at mid-anthesis using a hand-held mist sprayer. Mid-anthesis was considered as $50 \%$ of the spike had extruded anthers. For CMS lines, this stage was estimated by head size and the fertile alloplasmic line having extruded anthers. Each randomly chosen spike was sealed in a transparent plastic bag for 72 hours after spray inoculation. The bags ensured high humidity which is favorable for infection. There were three experiments in this research. For experiment 1, six spikes were selected per replication, eight replications for each cultivar; for experiment 2 , four spikes per replication and fif- 
teen replications for each cultivar; and for experiment 3, four to six spikes per replication and thirteen replications for each cultivar were used. Because there were multiple replications and multiple spikes per replication, each cultivar was inoculated on different dates within a 10 to 15 day period. Based upon the results with low infection from experiment $1\left(1.0 \times 10^{5}\right.$ spores $\mathrm{mL}^{-1}$ bagging for 72 hours), experiment 2 was conducted using $2.0 \times 10^{5}$ spores $\mathrm{mL}^{-1}$ and bagging the spikes for 72 hours, and experiment 3 was conducted using $2.0 \times 10^{5}$ spores $\mathrm{mL}^{-1}$ and bagging the spikes for 96 hours which was 24 hours longer inoculation comparing with experiment 2 .

Disease assessment was done 21 days after the plastic bags were removed (24 to 25 days after mid-anthesis) in all three experiments. Disease was visually assessed as disease severity (\%) which is the percentage of diseased spikelets on a spike (Hernandez Nopsa et al. 2014). The final disease severity for a given treatment was the average of disease severity values on all spikes assessed in that treatment.

Data were subjected to analysis of variance using the general linear models (GLM) procedure using SAS version 9.2 (SAS Institute, Cary, NC). Data from experiments were analyzed separately and then subjected to a combined analysis based on a homogeneous error variance among the experiments (Gomez and Gomez 1984; Steel et al. 1997). The least significant difference (LSD) test at $P=0.01$ was used to compare pairs of treatment means.

In addition, a contrast was modeled using disease severity in CMS 77(2) and Yanshi NO.9 vs. M 77(2) and M Yanshi NO.9. A contrast was also modeled using disease severity in the maintainer lines of 77(2) and the maintainer of Yanshi NO.9 vs. the unrelated restorer of R113. The first contrast was done to highlight the difference among alloplasmic lines which greatly differed in cytoplasm, anther health and extrusion, and seed set. The second contrast compared male fertile lines which differed in nuclear and cytoplasm genes.

\section{Results}

Analysis of variance identified that significant differences existed among the cultivars for Fusarium head blight severity in experiments 1 and 2 and over all three experiments. With the cultivars being significantly different, the over experiments contrasts were done to determine where those differences were (Tables 1 and 2). 77(2) was significantly more resistant than M 77(2) $(P<.0001)$ and R113 $(P<.0001)$. Yanshi NO.9 was significantly more resistant than R113 $(P=0.0002)$ but not significantly different from M Yanshi NO.9 $(P=0.1770)$ (Table 2). The contrast of CMS 77(2) and Yanshi NO.9 vs. R113 (restorer) indicated that the CMS lines were significantly more resistant to FHB than the restorer line $(P<.0001)$. The contrast of the maintainer lines of 77(2) and the maintainer of Yanshi NO.9 vs. R113 line was not significant $(P=0.9229)$.

Each experiment was also considered separately to study the effects of low, moderate, and high levels of infection (Fig. 1). In experiment 1, the disease severities of the five cultivars were under 0.1 except M 77 (2) which was about 0.13 (Fig. 1). Disease severity of the two CMS lines, 77(2) and Yanshi NO.9, was significantly lower than that of their 


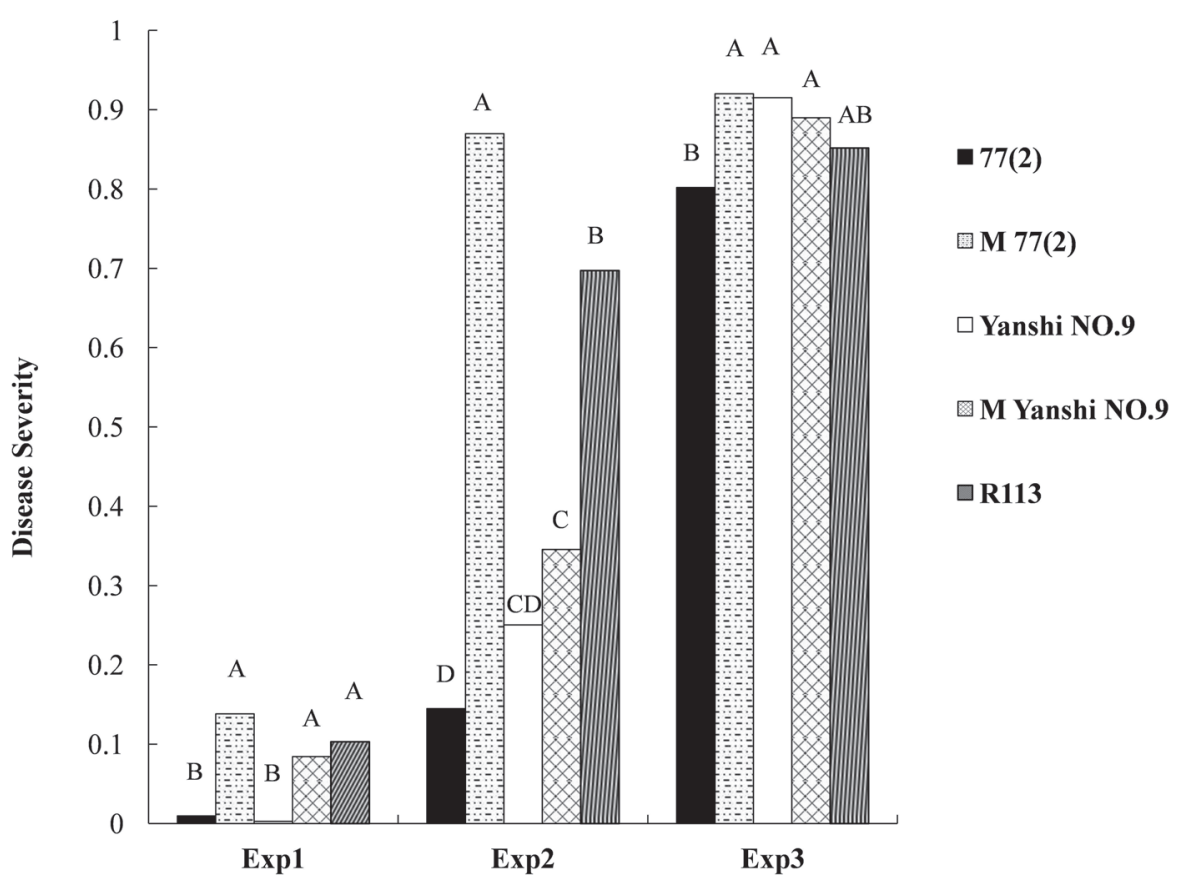

Figure 1. Average disease severity of five lines differing in their cytoplasm and male fertility inoculated in three experiments with different levels and for different durations with F. graminearum (isolate NE 90)

Means within each experiment followed by the same letter are not significantly different according to the least significant difference test at $P=0.01$

maintainer lines and the unrelated restorer line. In experiment 2 with twice the inoculum concentration, disease severity was higher than in experiment 1 as expected. 77 (2) was not significantly different from Yanshi NO.9, but significantly lower than M 77 (2), M Yanshi NO.9, and R113. Yanshi NO.9 was lower, but not significantly different from M Yanshi NO.9, but was significantly lower than M 77(2) and R113. In experiment 3, dis-

Table 1. Significant differences from Least Squares Means for Fusarium head blight disease severity among the two cytoplasmic male sterile lines, their alloplasmic maintainer lines, and a restorer line for all three experiments

\begin{tabular}{|l|c|c|c|c|c|}
\hline \multicolumn{1}{|c|}{$\mathrm{i} / \mathrm{j}$} & $77(2)$ & M 77 (2) & Yanshi NO.9 & M Yanshi NO.9 & R113 \\
\hline $77(2)$ & & $<.0001$ & 0.0857 & 0.0025 & $<.0001$ \\
\hline M 77 (2) & $<.0001$ & & $<.0001$ & $<.0001$ & 0.0213 \\
\hline Yanshi NO.9 & 0.0857 & $<.0001$ & & 0.1770 & 0.0002 \\
\hline M Yanshi NO.9 & 0.0025 & $<.0001$ & 0.1770 & & 0.0138 \\
\hline R113 & $<.0001$ & 0.0213 & 0.0002 & 0.0138 & \\
\hline
\end{tabular}


Table 2. Results from the General Linear Models procedure for comparing disease severity of two CMS lines, their alloplasmic maintainer lines, and a restorer line for all three experiments

\begin{tabular}{|l|c|c|c|c|}
\hline \multicolumn{1}{|c|}{ Parameter } & Estimate & $\begin{array}{c}\text { Standard } \\
\text { error }\end{array}$ & $t$-Value & $\operatorname{Pr}>|\mathrm{t}|$ \\
\hline $77(2)+$ Yanshi NO.9 vs. M 77(2) + M Yanshi NO.9 & -0.41 & 0.06 & $-6.57 * * *$ & $<.0001$ \\
\hline $77(2)+$ Yanshi NO.9 vs. R113 & -0.42 & 0.08 & $-5.46 * * *$ & $<.0001$ \\
\hline M 77(2) + M Yanshi NO.9 vs. R113 & -0.01 & 0.08 & $-0.10 \mathrm{~ns}$ & 0.9229 \\
\hline
\end{tabular}

*** significant at $P<0.001, \mathrm{~ns}=$ not significant $(P>0.05)$

ease severity was between 0.80 (77(2)) and 0.92 (M 77(2)). Differences among M 77(2), Yanshi NO.9, M Yanshi NO.9 and R113 in this experiment were minimal compared with the previous two experiments. However, 77(2) was significantly lower than M 77(2). Due to the higher level of inoculum and longer infection period, the disease overwhelmed the resistance of most of the cultivars which had similar levels of FHB infection.

\section{Discussion}

Artificial inoculation is a method that can be used to investigate general resistance to FHB. In this study, the CMS line of 77(2) with non-functional anthers was significantly more resistant than M 77(2) at all levels of FHB infection and generally lower than the cultivars with fertile anthers. Yanshi NO.9 was significantly lower than M Yanshi NO.9 at low levels of infection, however, at higher levels of infection, it was not significantly different from M Yanshi NO.9. These results indicated that the non-functional anther, the T. timopheevi cytoplasm, or the lack of seed reduced disease severity at low levels of infection and depending upon the nuclear background even at higher levels of infection. Anthers are considered to be the main site of initial or primary infections by $F$. graminearum (Shaner 2003). Therefore, the absence of anthers can confer resistance to initial infection. This resistance is one of two major categories of resistance to FHB: resistance to initial infection (Type I), and resistance to pathogen spread in infected tissue (Type II) (Shroeder and Christensen 1963). Our results are in agreement with those of Kubo et al. $(2010,2013)$ who found the severity of FHB infection in cleistogamous (closed flowering) recombinant inbred lines (RILs) and double haploid lines (DHLs) of wheat to be significantly lower than in chasmogamous (opened flowering) RILs and DHLs, hence demonstrating that the closed-flowering (no anther extrusion) characteristic was effective in increasing resistance to FHB infection. In their experiments, the lines set seed, hence seed set did not affect disease severity (as may have occurred in our experiments).

To consider the effect of the cytoplasm, we contrasted the maintainer lines with the unrelated restorer line (all lines have functional anthers, but differ in their cytoplasm and nuclear genes) and found that the three lines were not significantly different. In this case, the contrast could be explained by either: for lines with fertile anthers, the cytoplasm had no effect, or the genes in the nuclei of the three lines affected response due to the cytoplasm. Basically the results could also be explained if T. timopheevi cytoplasm is more resistant than T. aestivum cytoplasm, but the three lines had the same disease response if 
the restorer line had genes in the nucleus that increased the level of susceptibility so that the cytoplasmic resistance effects were reduced or masked by nuclear genes. The three lines all set seed, so the presence of seed may explain our results. The three fertile anther lines which set seed all had higher disease severity than the CMS lines which did not set seed, however, previous research by Kubo et al. $(2010,2013)$ indicated seed setting did not affect disease severity. Seed setting alone may not fully explain our results. In experiment 3 , the disease severity for all cultivars was from 0.80 to 0.92 . Hence the presence or absence of seed, the differences in cytoplasm, or the presence or absence of fertile anthers, did not greatly reduce the disease severity when the inoculum was higher and bagging period was longer. In experiment 3, the high disease pressure overwhelmed whatever resistance was found in experiments 1 and 2 which had low to moderate disease pressure. Experiment 3 also indicated that seeded cultivars and non-seeded cultivars can have the same result. It is possible that the observed higher disease severity at the higher inoculum concentration and longer bagging period was due in part to a lack or overwhelming of Type II resistance in all the cultivars. Using misted heads with high spore concentrations and long inoculation periods, could lead to so many Type I infections that the spread from these initial infection points (Type II resistance) had no effect. Based on the results in this study, we believe that non-extruded infertile anthers play an essential role in Type I FHB resistance in winter wheat especially at low inoculum concentrations. These result and conclusion agree with those of Matsui et al. (2002) who studied the effect of sterile and fertile anthers in CMS with and without restorer genes and in fertile euplasmic lines of barley. They are also consistent with the results of Kubo et al. (2010, 2013).

Our results are contrary to some previously published studies that showed that inoculated wheat spikes were invaded at other easily penetrable parts of the floret regardless of the anthers (Kang and Buchenauer 2000). This difference between our results and those of Kang and Buchenauer (2000) may be due to different lines being used, or to different disease protocols. The reason for significantly higher resistance in CMS cultivars with non-extruded infertile anthers compared with their corresponding maintainer and restorer is not known. Research by Miller et al. (2004) found that F. graminearum had high affinity to anthers and pollen. However, in 2010 Skinnes et al. reported that wheat genotypes with high levels of anther extrusion tended to develop less FHB and had lower levels of DON contamination. Hence, there may be multiple infection pathways, some of which are through anthers and others are not.

Based on these results, we conclude that the anther is most likely an important pathway of FHB infection. The disease severity showed significantly higher resistance in CMS lines though it varied with the genetic background and the inoculation protocol. Moreover, greater severity including Fusarium damaged kernels and the mycotoxin DON should be taken into account to characterize a cultivar as resistant or susceptible to FHB (Hernandez Nopsa et al. 2012). Differences in environmental conditions and periods of anthesis often hamper the interpretation of infection data with regard to cultivar resistance measured in the field (Doohan et al. 2003; Brennan et al. 2007; Cowger and Arrellano 2010). Further investigation is warranted on CMS, maintainer and restorer lines to deter- 
mine whether the resistance to FHB is caused by cytoplasmic differences, nuclear genes, or their interaction, as well as, how scab may affect future hybrid wheat production fields where CMS or chemical hybridizing agents will be used to create male sterile plants with shriveled or infertile anthers.

\section{Acknowledgements}

The present work was performed at the University of Nebraska, Lincoln, Nebraska, USA. We thank Prof. Gaisheng Zhang of Northwest A\&F University, Shaanxi, China for providing seeds. We also thank Janelle G. Counsell and graduate student Anita Panthi for helping setting inoculums, and Mitchell L. Montgomery, Gregory Dorn and Richard S. Little for all help in the greenhouse and Seed Lab. Partial funding for P.S. Baenziger is from Hatch project NEB-22-328, USDA-IFAFS competitive grant 2001-04462, USDA, NRICGP 00-353000-9266, and 2004-35300-1470, USDA, CSREES NRICAP grant numbers 2006-55606-16629 and 2011-68002-30029 (Triticeae-CAP), USDA OREI 2007-51300-03785, and USDA under Agreement No. 59-0790-4-092 which is a cooperative project with the U.S. Wheat \& Barley Scab Initiative. Any opinions, findings, conclusions, or recommendations expressed in this publication are those of the authors and do not necessarily reflect the views of the USDA. Finally, we thank China Scholar Council for supporting Mengyuan Wang's two years visit in the USA.

\section{References}

Audenaert, K., Van Broeck, R., Bekaert, B., De Witte, F., Heremans, B., Messens, B., Höfet, M., Haesaert, G. 2009. Fusarium head blight (FHB) in Flanders: population diversity, inter-species associations and DON contamination in commercial winter wheat varieties. Eur. J. Plant Pathol. 125:445-458.

Brennan, J.M., Leonard, G., Fagan, B., Cooke, B. M., Ritient, A., Ferracane, R. 2007. Comparison of commercial European wheat cultivars to Fusarium infection of head and seedling tissue. Plant Pathol. 56:55-64.

Brown-Guedira, G., Griffey, C., Kolb, F., Mckendry, A., Murphy, J.P., Van Sanford, D. 2008. Breeding FHBresistant soft winter wheat: progress and prospects. In: Proc. $3^{\text {rd }}$ Int. FHB Symposium Szeged, Hungary. Cereal Res. Commun., Suppl. B 36:31-35.

Cowger, C., Arrellano, C. 2010. Plump kernels with high deoxynivalenol linked to late Gibberella zeae infection and marginal disease conditions in winter wheat. Phytopathol. 100:719-728.

Cuthbert, P.A., Somers, D.J., Cloutier, S., Babel, A.B. 2006. Fine mapping Fhb1, a major gene controlling fusarium head blight resistance in bread wheat (Triticum aestivum L.). Theor. Appl. Genet. 112:1465-1472.

Dai, S.J. 2012. Cause analysis and countermeasures for FHB epidemic of wheat in Huaibei Area in 2012. J. Anhui Agri. Sci. 17:557-558. (in Chinese)

Doohan, F.M., Brennan, J., Cooke, B.M. 2003. Influence of climatic factors on Fusarium species pathogenic to cereal. Eur. J. Plant Pathol. 109:755-768.

Gomez, A.K., Gomez, A.A. 1984. Statistical Procedures for Agricultural Research. Second Ed. John Wiley \& Sons. New York, NY, USA.

Groth, J., Tamburic-Ilincic, L., Schaafsma, A., Brule-Babel, A., Hartl, L. 2011. FHB resistance of winter wheat from Canada and Europe estimated across multi-environments after inoculation with two deoxynivalenol producing fusarium species. Cereal Res. Commun. 39:189-199.

Hernandez Nopsa, J.F., Baenziger, P. S., Eskridge, K.M., Peiris, K.H.S., Dowell, F.E., Harris, S.D., Wegulo, S.N. 2012. Differential accumulation of deoxynivalenol in two winter wheat cultivars varying in FHB phenotype response under field conditions. Can. J. Plant Pathol. 3:380-389. 
Hernandez Nopsa, J.F., Wegulo, S.N., Panthi, A., Hallen-Adams, H.E., Harris, S.D., Baenziger, P.S. 2014. Characterization of Nebraska isolates of Fusarium graminearum causing head blight of wheat. Crop Sci. $\mathbf{5 4 : 3 1 0 - 3 1 7 . ~}$

Jones, R.K., Mirocha, C.J. 1999. Quality parameters in small grains from Minnesota affected by fusarium head blight. Plant Dis. 83:506-511.

Kang, Z.S., Buchenauer, H. 2000. Cytology and ultrastructure of the infection of wheat spikes by Fusarium culmorum. Mycol. Res. 104:1083-1093.

Kubo, K., Kawada, N., Fujita, M., Hatta, K., Oda, S., Nakajima, T. 2010. Effect of cleistogamy on Fusarium head blight resistance in wheat. Breeding Sci. 60:405-411.

Kubo, K., Fujital, M., Kawadal, N., Nakajima, T., Nakamura K., Maejima, H., Ushiyama, T., Hatta, K., Matsunaka, H. 2013. Minor differences in anther extrusion affect resistance to Fusarium head blight in wheat. J. Phytopathol. 161:308-314.

Liu., Q.X., Lillemo, M., Skinnes, H., He, X.Y., Shi, J.R., Ji, F., Dong, Y.H., Bjørnstad, A. 2013. Anther extrusion and plant height are associated with Type I resistance to Fusarium head blight in bread wheat line 'Shanghai-3/Catbird'. Theor. Appl. Genet. 126:317-334.

Matsui, K., Yoshida, M., Ban, T., Komatsuda, T., Kawada, N. 2002. Role of male-sterile cytoplasm in resistance to barley yellow mosaic virus and Fusarium head blight in barley. Plant Breed. 121:237-240.

McMullen, M., Jone, R., Gallenberg, G. 1997. Scab of wheat and barley: a re-emerging disease of devastating impact. Plant Dis. 81:1340-1348.

Miller, S.S., Chabot, D.M.P., Ouellet, T., Harris, L.J., Fedak, G. 2004. Use of a Fusarium graminearum strain transformed with green fluorescent protein to study infection in wheat (Triticum aestivum). Can. J. Plant Pathol. 26:453-463.

Nganje, W.E., Bangsung, D.A., Leistritz, F.L., Wilson, W.W., Tiapo, N.M. 2004. Regional economic impacts of fusarium head blight in wheat and barley. Rev. Agri. Eco. 26:332-347.

Parry, D.W., Jenkinson, P., Mcleod, L. 1995. Fusarium ear blight (Scab) in small-grain cereals: a review. Plant Pathol. 44:207-238.

Pugh, G.W., Johann, H., Dickson, J.G. 1933. Factors affecting infection of wheat heads by Gibberella saubinetti. J. Agri. Res. 46:771-797.

Schroeder, H.W. 1955. Factors affecting resistance of wheat to scab caused by Gibberella zeae (Schw.) Petch. $\mathrm{PhD}$ thesis. University of Minnesota. St Paul, MN, USA.

Schroeder, H.W., Christensen, J.J. 1963. Factors affecting resistance of wheat to scab by Gibberella zeae. Phytopathol. 53:831-838.

Shaner, G. 2003. Epidemiology of Fusarium head blight of small grain cereals in North America. In: Leonard, K.J., Bushnell, W.R. (eds), Fusarium Head Blight of Wheat and Barley. Am. Phytopathol. Soc. St. Paul, MN, USA. pp. 84-119.

Skinnes, H., Semagn, K., Tarkegne, Y., Maroy, A. G., Bjornstad, A. 2010. The inheritance of anther extrusion in hexaploid wheat and its relationship to Fusarium head blight resistance and deoxynivalenol content. Plant Breed. 129:149-155.

Song, J., Hedgcoth, C. 1993. Influence of nuclear background on transcription of a chimeric gene (orf 256) and cox1 in fertile and cytoplasmic male sterile wheats. Genome 37:203-209.

Steel, R.G.D., Torrie, J.H., Dickey, D.A. 1997. Principles and Procedures of Statistics. A Biometrical Approach. Third Ed. McGraw-Hill. New York, NY, USA.

Strange, R.N., Smith, H. 1971. A fungal growth stimulant in anthers which predisposes wheat to attack by Fusarium graminearum. Physiol. Plant Pathol. 1:141-150.

Strange, R.N., Majer, J.R., Smith, H. 1974. The isolation and identification of choline and betaine as the two major components in anthers and wheat germ that stimulate Fusarium graminearum in vitro. Physiol. Plant Pathol. 4:277-290.

Tu, C. 1930. Physiologic specialization in Fusarium spp. causing head-blight of small grains. Minnesota Agricultural Experimental Station. Technical Bulletin 74:1-27.

Tomasovic, S., Miinar, R., Ikic, I., Katarina, J., Palaversic, B. 2007. Breeding wheat for resistance to Fusarium head blight. Cereal Res. Commun. 35:1209-1212. 
Wilson, J.A., Ross, W.M. 1962a. Cross breeding in wheat (Triticum aestivum) hybrid seed set on a cytoplasmic male-sterile winter wheat composite subjected to cross pollination. Crop Sci. 2:415-417.

Wilson, J.A., Ross, W.M. 1962b. Male sterility interaction of the Triticum aestivum nucleus and Triticum timopheevi cytoplasm. Wheat Inf. Serv. 14:29-30.

Zhang, Z.H., Zhang, X.K., Sun, D.J., Wang, X.D., Fu, X.J. 2009. Distribution of vernalization and photoperiod genes in Shaanxi wheat cultivars. J. Triticeae Crops 3:401-408. (In Chinese with English abstract) 